\title{
REDES DE ACCIÓN PÚBLICA EN LA GESTIÓN LOCAL: TENDENCIAS ACTUALES
}

\section{Suzana Moura*}

Este artículo discute los cambios que están ocurriendo en la gestión local en Brasil, en las dos últimas décadas, tanto en términos de ideas como en la práctica. A partir de la identificación de movimientos que aparecen como propulsores de innovación, se analiza lo que serían algunas de las tendencias presentes en los años 90 y, más específicamente, en lo que concierne a las redes de interacción gobierno-sociedad. Se trata de un segmento de una investigación que está en desarrollo, con vistas a una tesis doctoral.

\section{LO QUE HAY DE NUEVO EN LA GESTIÓN LOCAL}

La (re)valorización del espacio local y las nuevas directrices de la gestión pública en ese ámbito estuvieron asociadas, en los años 70 y 80 , a los procesos de descentralización del Estado que tuvieron lugar en Europa y América Latina, lo que en algunos países se articuló con los movimientos de democratización. Una de las cuestiones centrales de aquel momento se refería a la afirmación de un poder local con cierto grado de autonomía o, por el contrario, a la negación de esa posibilidad'. Las posibilidades de ampliación de la democracia, cuestión relevante, se traducía en términos de participación popular y descentralización intraurbana.

Si en ese período el énfasis del debate recaía sobre la dimensión política de la gestión local -en términos de las relaciones con el gobierno central, por un lado, y de las relaciones con la sociedad, por otro- en los años 90 el foco parece dirigirse hacia la dimensión económica de las estrategias de desarrollo local. En este caso, lo local aparece interactuando más allá del ámbito nacional e internamente se ponen de manifiesto relaciones distintas a las enfatizadas en el período anterior. La integración competitiva en el mercado mundial, por un lado, y la constitución de ámbitos y foros plurales de negociación, por el otro, se colocan como expresiones de las nuevas coordenadas para la gestión pública local. Tal movimiento no se percibe únicamente como respuesta a las crisis y reestructuraciones que ocurren en el Estado, referidas fundamentalmente al proceso de reconfiguración productiva, al reemplazo del fordismo por la acumulación flexible ${ }^{2}$.

A partir del examen de la literatura referida al fenómeno citado -revalorización de lo local y de la gestión pública en ese

\footnotetext{
Traducción del portugués: Ana Inés López-Accotto.

(*) Profesora de la Escuela de Administación de la UFBA y doctoranda de la NPGA/UFBA. (1) Sobre ese debate, ver Massolo (1988), Pavía y Ospina (1992), Arocena (1989), Coraggio (1988) Botero (1992), Daniel (1988), Castro (1988), Biarez ( ), Fischer et al (1993), Moura (1993).

(2) Ver Harvey (1992), Storper (1991, 1994), Syret (1993).
}

ámbito a partir de los años 70- es posible configurar dos movimientos propulsores de innovación que están presentes en el escenario brasileño, y que se abordan aquí como activismo democrático e iniciativas empresariales urbanas.

\section{Activismo democrático}

El denominado activismo democrático remite a ideas y prácticas de gestión pública que emergen en Brasil en el seno de la transición democrática, que surgen como fruto de la confluencia más o menos directa de las acciones de movimientos sociales y de gobiernos municipales dirigidos por sectores de izquierda y progresistas (Leal, 1994; Daniel, 1990, 1992 y 1994; Moura, 1993 y 1994; Caccia-Bava, 1994; Kowarick y Singer, 1993; Pacheco, 1993)

La idea de constitución de un "ideario democrático" de la gestión pública sirvió para caracterizar a ese movimiento que se remonta a mediados de los años $70^{3}$, con las conocidas experiencias de Lages (SC), Piracicaba (SP) y Boa Esperança (ES) y pasa por las acciones colectivas como el referente de la reforma urbana, retomándose en gobiernos de centros urbanos de tamaño medio y grande en los años 90 -los casos de Sao Paulo, Porto Alegre y Santo André, por ejemplo.

La participación popular en los procesos de decisión -o ciudadanos-, la democratización de las informaciones y del acceso a los bienes y servicios públicos, el rescate de la autonomía y de la identidad local y la inversión de las prioridades, son expresiones que sintetizan ese ideario (Daniel, 1990; Moura, 1993; Leal, 1994). La idea de inversión de prioridades es utilizada para contraponerla a lo que sería la agenda pública tradicional, caracterizada por la prioridad dada a las grandes obras que atienden poco a las demandas populares. Al mismo tiempo, se integró explícitamente en la agenda pública un aspecto nada material: el cambio en el plano de la cultura política que estaría asociado a la construcción de nuevas formas de interacción entre gobierno, administración pública y sociedad. La participación popular en las decisiones y la transparencia político-administrativa rompería, en este punto, con los patrones tradicionales caracterizados por el acceso oscuro y selectivo de las élites locales a los centros de decisión y, en contrapartida, el distanciamiento de sectores populares, caracterizado por las relaciones clientelares.

Esas ideas pasan por redefiniciones, con el transcurrir del tiempo y los desdoblamientos de las experiencias. Por ejemplo,

(3) Leal (1994) al tratar sobre ese ideario, rescata como antecedentes, las experiencias de gobiernos progresistas desarrolladas en los años 50/60 en Pernambuco. 
en las primeras experiencias, hasta el final de los años 80, el énfasis de la democratización de la gestión pública recaía en los sectores populares y sus organizaciones. Ya en los años 90, se observa una tendencia de ampliación de tal postura, hacia el conjunto de la sociedad, incluidos los agentes económicos, cuya relación en el período anterior era marcadamente conflictiva. Dicho cambio, además de representar una ampliación en el espectro de los actores y segmentos sociales, incide en el propio sentido atribuido a la idea y a las prácticas de participación, que incorpora las dimensiones de negociación, cooperación y asociación (Moura, 1993).

Ese cambio guarda relación con un conjunto de factores: la profundización de la crisis económica y de la recesión; el reflujo y la pérdida de visibilidad política de los movimientos sociales; y la propia madurez de las experiencias, principalmente cuando éstas tienen lugar en centros urbanos -cuya complejidad de gestión local es mucho mayor que en municipios pequeñosenfrentándose a una mayor diversidad de intereses y a una mayor interdependencia con la iniciativa privada. En ese contexto se amplía también la agenda pública local con la incorporación de la cuestión económica ${ }^{4}$. A diferencia de la iniciativa empresarial, como se abordará más adelante, el énfasis mayor no está en la búsqueda de la competitividad sino en la garantía de niveles satisfactorios de empleo, de gestión y de distribución de la rentas.

¿Cuál sería el elemento demarcador y conformador de ese ideario, que se traduce en activismo democrático? Para Guimaraes y D'Arc (1994) se trata de "principios morales y de justicia social". Para Leal (1994) y Daniel (1994) se trata de una revisión de paradigmas en el campo de la izquierda frente a la quiebra del socialismo real y de referencias anteriores, en las que se afirma la democracia como un "valor estratégico" -o lo que algunos llaman "democracia radical". Esos y otros elementos que se sitúan en el campo político-filosófico merecen ser tomados en consideración.

\section{Iniciativa empresarial urbana}

Así como el activismo democrático está relacionado, más directamente, con los cambios en el plano de la política local, la iniciativa empresarial urbana sirve para indicar un movimiento de redefinición del papel y actuación de los gobiernos locales en lo tocante a la economía, asociado a las ideas de búsqueda de eficiencia de la gestión urbana de cara a la integración competitiva en el mercado global (Harvey, 1989; Borja, 1994; Osborne y Gaebler, 1993; Peñalva y Finquelievech, 1993; Berry, 1994; Syret, 1993; Hamel, 1990; Pacheco, 1993).

De acuerdo con Harvey (1989), refiriéndose a ciudades europeas y americanas, existe una tendencia de alteración del

(4) Se pueden encontrar iniciativas en ese campo desde las primeras experiencias. Pero entonces se desarrollaban básicamente en municipios pequeños en los que tiene relevancia la problemática de los pequeños productores y trabajadores rurales. En los centros urbanos se tomaba en consideración la cuespequenos productores y trabajadores rurales. En los centros urbanos se tomaba en consión
tión del control del uso y ocupación del suelo y de la provisión de bienes y servicios.

(5) Son ejemplos ilustrativos: las acciones en busca de alternativas a procesos de despidos masivos de trabajadores y de privatización de empresas públicas, que tuvieron lugar en varios municipios en el período 89-92, entre los cuales pueden señalarse Santos/SP, Angra dos Reis/RJ e Ipatinga/MG; la creación de foros integrando a agentes económicos, sindicatos de trabajadores y otras organizaciones, en el intento de buscar alternativas a la crisis y a la polícita recesiva también en el mismo período, como los ejemplos de Porto Alegre/RS y Santo André (Moura, 1993). papel de los gobiernos locales, que estarían adoptando una postura menos "gerencialista" -dedicada a la provisión de servicios y beneficios para la población- con el énfasis dado, a partir de los años 80, a la exploración de nuevos medios de desarrollo económico. Lo que el autor denomina como nueva iniciativa empresarial urbana tiene que ver con el estilo de gobierno que se estaría experimentando para explorar esos nuevos medios: el gobierno asume un papel de facilitador y coordinador de un conjunto de fuerzas movilizadas por agentes sociales diversos; y la formación de asociaciones público-privado como base para nuevas inversiones o atracción de fuentes de financiación externas. Para asegurar ventajas comparativas, en un cuadro de estimulación de la competitividad urbana, serían esenciales la reorganización de la vida urbana siguiendo esos modelos y la transformación de la ciudad en un gran negocio. La ciudad debe adquirir el espíritu de empresa, como dicen Peñalva y Finquelievech (1993).

Borja (1994) se refiere a ese mismo movimiento abordando lo que denomina protagonismo de las ciudades o del gobierno local y, adoptando una postura prescriptiva, afirma que tal protagonismo estaría garantizado con una promoción de la ciudad hacia el exterior, a través de una imagen fuerte y positiva basada en ventajas comparativas, y su promoción interna, buscando reforzar el "espíritu cívico" entre sus habitantes. Además de la asociación entre lo público y lo privado, el autor sitúa la participación ciudadana como elemento clave del nuevo estilo de intervención gobierno-sociedad.

Aunque reflexionando sobre la temática de las nuevas formas de gestión pública, en su sentido amplio, Osborne y Gaebler (1993) señalan algunos elementos para el debate que se suman a los tratados anteriormente. Para estos autores los gobiernos locales estarían respondiendo con más énfasis a los requisitos del nuevo patrón de acumulación, adoptando políticas alternativas para la prestación de servicios -asociaciones entre lo público y lo privado en la financiación y ejecución, estímulo a la competencia entre prestadores de servicios y el enfoque en la calidad y en la adopción de la gestión pública requerida en la era de la acumulación flexible. La referencia a innovaciones practicadas en el mundo de las empresas privadas para la organización de los servicios públicos y de las estrategias de desarrollo económico local, es un aspecto resaltado por otros autores (Peñalba y Finquelievech, 1993 y Berry, 1994).

A partir del debate sobre desarrollo local, Syret (1993) y Hamel (1990), destacan rasgos semejantes para indicar lo que sería la perspectiva liberal para el primero o elitista, para el segundo. Estas se diferenciarían de otras aproximaciones más próximas al activismo democrático, denominadas radical y social, respectivamente (Loiola y Moura, 1994). Independientemente de las diferenciaciones, estas aproximaciones reflexionan sobre un mismo fenómeno: la difusión del ideario y de un conjunto de prácticas que enfatizan la dimensión emprendedora de la gestión local, en el sentido de la búsqueda de eficiencia y efectividad económica. Ciertamente, la calidad de la vida urbana se convierte en un elemento esencial para volver más competitiva la ciudad.

El debate sobre la iniciativa empresarial local sólo recientemente empieza a ser visualizado y abordado en América Latina. Borja, por ejemplo, observa que los procesos de democratización y de descentralización que tuvieron lugar en diversos países latinoamericanos en la década de los años 80 , revalorizaron el papel de las ciudades y de los gobiernos locales, pero sin lle- 
gar a implicar un avance hacia su protagonismo. Protagonismo que, para el autor, significa la adopción de una postura emprendedora en las formas señaladas por Harvey. En ese sentido, Borja considera que un avance en esa dirección tiene lugar en los años 90 con la adopción de iniciativas que buscan la elaboración y ejecución de planes estratégicos en ciudades tales como Porto Alegre, Río de Janeiro, Bogotá y Córdoba, a semejanza de lo ocurrido en Barcelona ${ }^{6}$.

Pacheco (1993 y 1994), adoptando las referencias de Harvey, procura identificar la emergencia de la nueva iniciativa empresarial urbana en ciudades brasileñas, entre las cuales sitúa la experiencia del consorcio intermunicipal del $\mathrm{ABC}$ paulista, iniciada en 19917. Siguiendo la misma línea que Pacheco, Pinhanez et al. (1993) y Silva et al. (1993), desarrollaron estudios que identificaban la experiencia de Curitiba, a partir de los gobiernos de Jaime Lerner -71/75, 79/82 y 89/92- como representativa de la iniciativa empresarial urbana ${ }^{8}$.

En las aproximaciones sobre los movimientos de iniciativa empresarial urbana y de activismo democrático, se destacan tres planos fundamentales que expresarían lo que hay de nuevo en la gestión local: la agenda pública -el acento dado a la cuestión de la economía local, de la ciudad como negocio y de la ciudadanía/democracia; el papel del gobierno local -catalizador/articulador de fuerzas, emprendedor y activista político; y las formas de interacción producidas -participativas, negociadoras, compartidas. Importa, entonces, discutir las convergencia y diferenciaciones en esos años 90.

\section{TENDENCIAS DE LOS AÑOS 90}

Considerando los dos movimientos citados, se observa una tendencia de refuerzo de algunos de los elementos aportados por la iniciativa empresarial urbana, inclusive en el seno de las experiencias identificadas como el primer movimiento. Uno de los factores importantes de convergencia se encuentra en el hecho de que la dimensión económica se vuelve un punto central de la agenda pública local, con una reproducción de experiencias semejantes en asociaciones con sectores capitalistas, que busca una ampliación de la renta local y la atracción de nuevas inversiones $^{9}$. Tal hecho puede observarse en situaciones distintas, tales como la difusión reciente del modelo de plan estratégico de Barcelona en ciudades latinoamericanas -Río de Janeiro, Salvador, Córdoba, Bogotá, entre otras-, las nuevas iniciativas desarrolladas por el gobierno del Frente Popular en Porto Alegre y el fortalecimiento de la perspectiva de marketing de la ciudad de Curitiba.

(6) El modelo de planificación estratégica construido en Barcelona a final de los años 80 es una expresión de inciativa emprendedora local y ha sido difundido y aplicado en algunas ciudades brasileñas. (7) Se trata de una iniciativa de gobiernos municipales de la región para tratar problemas comunes, entre ellos el impacto de la crisis económica en la región, involucrando a los alcaldes, sectores empresariales, de sindicatos de trabajadores y de movimientos ambientalistas, entre otros (Pacheco, 1993).

(8) Los autores destacan el aspecto del desarrollo de ventajas comparativas y las estrategias de marketing adoptadas, como expresión de ello. $\mathrm{O}$ sea, la ìcapital de la planificación urbanaî y, posteriormente, la icapital ecológicaî se volvieron imágenes difundidas y símbolos de innovación en la gestión local

(9) Es ilustrativo el debate iniciado por Tarso Genro -alcalde de Porto Alegre- en el artículo "Por una política internacional de las ciudades"-diario Folha d: Sao Paulo. Genro destaca el papel de las ciudades en el incremento del comercio y del cambio de tecnalogías en el plano internacional, con otras ciudades. sin una intermediación del gobierno central, en función de una estrategia de inserción en el "nuevo orden". Del mismo modo es ilustrativa la constitución en Porto Alegre de un foro que implica a agentes económi cos locales, entre otros, en la búsqueda de alternativas de desarrollo, a partir del cual se desarrolló, por ejemplo, un proyecto de incubadora tecnológica reuniendo a varios socios.
La experiencia del plan estratégico "Barcelona 2000”, iniciada hacia 1988, trae algunos de los rasgos característicos de la iniciativa empresarial. Como objetivos básicos, la búsqueda de la integración en Europa y de la competitividad internacional. El marketing de la ciudad, su promoción externa e interna, se constituye en uno de los elementos clave del modelo, además de la comunicación y amplia articulación del gobierno local con las organizaciones privadas, populares y públicas, en foros de consulta y negociación. Esta ha sido una referencia importante y su difusión corrió, inclusive, por cuenta de una consultoría llevada por profesionales de Barcelona.

El objetivo del plan "Bogotá 2000" es una transformación de la ciudad en una metrópoli emprendedora, asentada en una nueva gobernabilidad y con mejor calidad de vida. El plan de Río de Janeiro es convertir la ciudad en una metrópoli con calidad de vida, asentada en la competitividad, en el respeto a la ciudadanía y a la cosa pública. Con sus particularidades, las dos ciudades llegan a propósitos y adoptan estrategias de construcción del plan semejantes.

El caso de Porto Alegre se destaca, en primer lugar, por la continuidad del gobierno, que desde 1989 está bajo la dirección del PT (Partido de los Trabajadores). La inversión en una línea de democratización aparece ya en 1989, siendo ilustrativa la iniciativa adoptada de discusión pública del presupuesto municipal -el conocido "Presupuesto participativo". En 1993, en el segundo mandato, surje el proyecto "Ciudad Constituyente", que busca ampliar el ideario de construcción de nuevas esferas públicas, teniendo en la mira el pensar y realizar colectivamente un proyecto estratégico de ciudad, donde se incluyese el desarrollo económico. Acciones como la instalación de una incubadora tecnológica y el intercambio con ciudades de países centrales se insertan en esa nueva etapa de gobierno.

Por su parte, la ciudad de Curitiba está construyendo desde los años 70 estrategias de desarrollo local a partir de un liderazgo que estuvo en tres períodos al frente del Ayuntamiento (71/75, 79/82 y 89/92), ganando su sucesor las elecciones de 1992. El desarrollo de estrategias de ampliación y cualificación de la infraestructura de los servicios urbanos, unidas a un trabajo importante de marketing de la ciudad -destinado, entre otras cosas, a atraer inversiones privadas- difundieron a Curitiba como la "ciudad de la planificación" y, más recientemente, como la "ciudad ecológica". Aunque no se observa el mismo proceso público y compartido de Barcelona, las estrategias adoptadas van en la dirección de hacer a la ciudad más competitiva en el escenario nacional e internacional.

Estos son algunos de los ejemplos sobre los cuales es interesante profundizar el estudio de las convergencias y diferenciaciones del activismo democrático y de la iniciativa empresarial urbana en un momento de refuerzo de la segunda. Ciertamente, examinarlos como movimientos distintos puede llevar a la dicotomización de un proceso que tiene intersecciones. El ideario de ampliación de la democracia, que es expresión del primero, tiende a ser contrapuesto al neoliberalismo visto como una vertiente que también estaría forjando cambios en el plano de la gestión local, como las políticas de desmonte del welfare state ${ }^{10}$ (Hamel, 1990; Daniel, 1992; Syret, 1993; Moura, 1993; Leal, 1994). El 
diseño de estrategias de desarrollo económico buscando la integración competitiva en el mercado mundial, al aparecer de modo exclusivo o incluso privilegiado en la agenda local, estaría más próximo a esta última vertiente.

En cambio, conforme se ha visto, es posible encontrar algunas convergencias de la iniciativa empresarial urbana con experiencias identificadas con el activismo democrático. Por otro lado, la inserción de la calidad de vida urbana como estrategia de desarrollo de ventajas comparativas -como ocurre en Curitibapuede implicar la democratización del acceso a la infraestructura y los servicios urbanos de capas más amplias de población.

De la misma forma que las convergencias es importante analizar las diferencias que justifican el tratamiento como dos movimientos y no como expresiones de un mismo movimiento que se sucederían en el tiempo, volviendo casi indistintas las perspectivas adoptadas (Pacheco, 1993; Levy, 1992). Un punto de partida para ese análisis es la consideración del factor político-ideológico como un aspecto que lleva a diferenciaciones entre el activismo democrático y la iniciativa empresarial urbana.

La cuestión de la ampliación de la ciudadanía en términos políticos y económicos y, dentro de ésto, la idea de construcción de una nueva cultura política, si se integraran en la agenda pública local como objetivos compartidos por el gobierno y otros actores sociales, puede llevar a formas de integración y políticas de desarrollo diferenciadas de aquellas producidas en un contexto cuya orientación hegemónica es el desarrollo de estrategias que vuelvan más competitivas a las ciudades y a los segmentos económicos instalados en ellas. En el caso de Porto Alegre, por ejemplo, la cuestión de la integración competitiva parece sustituirse por la idea de la búsqueda de una modernidad que implique una "democratización radical" y la integración de los excluidos.

Centrándose en las formas de interacción producidas entre el gobierno y la sociedad civil, en el contexto de experiencias representativas de esos movimientos, es interesante investigar, además de las diferenciaciones y convergencias, en qué medida han tenido lugar innovaciones frente a los modelos tradicionales.

\section{NUEVAS Y ANTIGUAS REDES DE ACCIÓN PÚBLICA}

Al observar experiencias como las citadas anteriormente, se identifica la (re)producción de redes tradicionales, como las clientelares, y nuevas redes plurales y públicas. Se distingue aquí la noción de red como metáfora, para indicar un modo de aprehender la dinámica de los procesos interactivos entre actores y organizaciones ${ }^{11}$, de aquella utilizada como expresión de una tendencia reciente de incremento de nuevas composiciones interorganizacionales.

Tomando la segunda perspectiva, se destaca una serie de conceptos de redes: redes sistémicas (Alter y Hagg, 1993), redes de innovadores (Freeman, 1991), redes estratégicas (Randolph, 1994), redes políticas (Miller, 1994) y redes de inserción local

(11) Tales procesos interactivos pueden ser caracterizados por una cierta regularidad, por su carácter más o menos formal, y por estar basados en propósitos comunes o complementarios. El carácter y la diná mica en cada contexto va a depender de las organizaciones y actores implicados, de sus propósitos y de las mica en cada contexto va a depender de
condiciones objetivas en que se sitúan.
(Mauriel, 1991), entre otras ${ }^{12}$. A pesar de las diferencias contenidas en esas expresiones, ellas apuntan a un tipo de articulación en red que se da entre organizaciones diversas -sean estatales, de esferas de gobierno diferenciadas o no, empresas de tamaños y actividades distintas, ONGs y/o movimientos sociales o grupos de otra naturaleza- teniendo como objetivos proyectos o políticas delimitados, asentados en relaciones más o menos formales y regulares (Loiola y Moura, 1994).

A modo de ilustración, se rescatan algunas definiciones que abordan más directamente el campo de la acción pública. Dentro de la idea de coordinación y movilización de recursos, Alter y Hage (1993) definen las redes sistémicas como un agrupamiento de organizaciones que toman decisiones conjuntas e integran esfuerzos para producir un bien o servicio ${ }^{13}$. Una de las referencias empíricas, en este caso, son los procesos de articulación entre agencias gubernamentales y organizaciones comunitarias en el plano local, en los Estados Unidos, que buscan la prestación de servicios. Aquellos procesos que se siguieron en el desmantelamiento de políticas sociales en el gobierno de Reagan. En una línea semejante y con referencia a la experiencia francesa, Maurel (1991) observa que las redes de inserción local significan una recomposición de las políticas sociales y del papel del Estado y de las "colectividades locales", permitiendo la articulación de los diversos actores e iniciativas presentes en el Estado y en la Sociedad Civil, en torno a una acción pública.

Con una preocupación no tan volcada a la cuestión de la movilización de recursos como los dos autores anteriores, Miller (1994) destaca el concepto de red política como un proceso de interacciones regulares, pero no institucionalizadas, que comprende al conjunto de los actores interesados e implicados en una política pública, constituyendo una arena para el debate, argumentación, escucha y juicio moral de la políticas. De ese modo, la red política sería un camino para procesar divergencias y conflictos. La referencia empírica del autor son las experiencias de formulación e implantación de planes locales dirigidos al problema de la protección ambiental en ciudades americanas.

Sin asumir las proposiciones implícitas en los conceptos señalados más arriba, los considera como indicativos de formas de interacción gobierno-sociedad que pueden desarrollarse en el plano local como expresión de la iniciativa empresarial urbana y del activismo democrático. Pero estos serían tipos de red interorganizacional, entre otros que pueden hallarse.

Podrían ser consideradas, en principio, como nuevas las redes políticas de las que habla Miller, que se asemejan a redes socio-gubernamentales que emergen del activismo democrático -por su carácter de espacio público de negociación y formulación de políticas y de espacio plural, en términos del espectro de organizaciones y actores implicados (Moura, 1993). Redes de ese tipo pueden encontrarse en Porto Alegre, por ejemplo. Estas difieren de las redes tradicionales de clientela u otras, caracterizadas por la existencia de mecanismos informales y oscuros de acceso de las organizaciones y segmentos privilegiados a los centros de decisión (Loiola y Moura, 1994).

(12) Se destacan aquí las denominaciones de red que son presentadas como nuevas tendencias referidas a los campos de los negocios y de la acción pública. Otras denominaciones pueden encontrarse en la literatura sobre el tema, con referencia, inclusive, a las articulaciones entre ONGs y movimientos sociales, por ejemplo, redes de solidaridad (Randolph, 1994) y redes de movimiento (Scherer-Warren, 1994). Hay otros estudios de redes interorganizacionales, que enfatizan lo que sería el potencial de ese tipo de composición -ganar competitividad y efectividad, sin ninguna adjetivación (Provan y Milward, 1995; Ring, 1995): Al respecto de las distintas aproximaciones, ver Loiola y Moura (1994) y Scherer-Warren (1994).

(13) Los autores desarrollan ese concepto por referencia a composiciones interorganizacionales producidas también en el campo de los negocios. 
Las redes sistémicas de las que hablan Alter y Hage (1993) y las redes de inserción local mencionadas por Mauriel (1991), que van en el sentido de la movilización de recursos e implementación de políticas, implicando a un conjunto variado de organizaciones estatales y de la sociedad civil, pueden ser consideradas, en un primer momento, semejantes a las acciones de asociación y consorcios presentes en Barcelona y en Río de Janeiro, por ejemplo. Eso teniendo en cuenta los patrones convencionales de departamentalización y de integración vertical en la gestión de políticas públicas.

\section{CONCLUSIÓN}

Además de los patrones de interacción mencionados con anterioridad, se podrían identificar otros que indican innovaciones o que son simples reproducciones de algo ya constituido. Pero, en todos los casos, para que se pueda avanzar en la comprensión de la naturaleza y la dinámica de las redes que se constituyen en el plano de la gestión pública local, en el interior de los dos movimientos citados, y para aprehender las señales de cambio, es necesario analizarlas a la luz de referencias teóricas acerca de las formas de intermediación Estado-Sociedad Civil. O sea, se vuelve fundamental verificar en qué medida las redes políticas, las redes sistémicas y las redes sociogubernamentales reproducen o se distinguen de los patrones corporativistas y pluralistas de intermediación de intereses.

Con esa cuestión, que requiere un trabajo de investigación más minucioso, se puede profundizar el conocimiento sobre la novedad de lo que se está produciendo y en qué dirección, dando mayor consistencia al análisis de las redes de acción pública que se insertan en las perspectivas de la iniciativa empresarial urbana y del activismo democrático, en sus convergencias y diferenciaciones.

\section{REFERENCIAS BIBLIOGRÁFICAS}

Alter, G. e Hage, J. (1993), Organizations Working Togheter. Ludus, Sage.

Assessing Public-Private Interorganization Network.. (1995), Anais Workshop International Network. Jouy-en-Josas, European Science Foundation Programe.

Arocena, José. (1988), Los Paradigmas del Desarrollo y lo "local". Cuadernos del CLAHE, Montevideo, CLAHE, (41): 5-21.

_ (1992), Evaluación del Impacto de Proyectos en el Desarrollo Local. Montevideo, CLAEH, mimeo.

_ "Desenvolvimento Local na América Latina: Debates e Referentes Teóricos". Palestra proferida no V Colóquio sobre Poder Local, Salvador, NPGA/EAUFBa, (mimeo).

BATLEy, R. e STOKER, G. (Org.) (1991), Local Government in Europe: Trends and Devellopments. Londres, Macmillan Education Ltda.

BerRy, Frances S. (1994), "Innovation in Public Administration: the adoption of Strategic Planning”. Public Administration Review. Washington, American Society for Public Administration, (4):.... Jul/aug 1994.

BITTAR, Jorge (org.). (1992), O Modo Petista de Governar. São Paulo, Cadernos Teoria e Debate.

BorJA, Jordi. (1994), Las Ciudades y el Planeamiento Estratégico: una reflexión Europea e Latinoamericana. Barcelona, (mimeo). (1994), Estrategias de Desenvolvimento Local: a experiencia de Barcelona. Palestra proferida no VI Colóquio sobre Poder Local, Salvador, NPGA/EAUFBa, 1994 (em fita).

_ e ForN, Manuel. (1992), "Políticas de Europa y de los Estados para las Ciudades". Barcelona, Estudios Territoriales, (39): mai/ago.
Botero, D. (1992), Descentralización y Neoliberalismo: Balance de un Proceso. Bogotá, Fondo Editorial, CEIR.

Caccia-Bava, Silvio. (1994), "Trocando a Riqueza de Maos: Distribuição de Renda e Poder nas Pequenas Cidades". Revista Proposta, Rio de Janeiro, FASE, (54):12-16.

_ (1994), "Experiencias de Desenvolvimento Local". Palestra proferida no Fórum Regional de Administrações Municipais Democráticas, Natal, Fórum Nacional sobre Participação Popular, mimeo.

CAmpos, E. (1992), "Longe das Capitais". São Paulo, Revista Teoria e Debate, Partido dos Trabalhadores, (18): 7-15, out/dez.

Castro, M. H. "Descentralização e Política Social no Brasil: as Perspectivas dos Anos 90”. São Paulo, Espaço e Debates, NERU, (32).

Coraggio, J. L. (1988), "Poder Local, Poder Popular”. Montevideo, Cuadernos del CLAHE, CLAHE, (43/46): 101-120, 1988.

DAniel, Celso. (1990), "As Administrações Democráticas e Populares em Questão”. Espaço e Debates. São Paulo, NERU, (30): 11-27.

_ (1988), "Poder Local no Brasil Urbano". São Paulo, Espaço e Debates, NERU, (24): 26-39.

(1992), "Poder Local e Desenvolvimento". Palestra proferida no V Colóquio sobre Poder Local, Salvador, NPGA/EAUFBA, mimeo. (1994), "Gestão Local e Participação da Sociedade". VILLASBOAS, R. (Org), São Paulo, Publicações POLIS, (14).

Deroy-Pineau, Françoise. (1991), "Reseaux Sociaux et Mobilisation de Ressources". Revue Pour, Paris, (132): 119-130.

FASE (VVAA). (1990), Administraçôes Populares. Revista Proposta, Rio de Janeiro, FASE, (45)

(1992), "Democratizando a Cidade". Revista Proposta, Rio de Janeiro, FASE, (54).

Ferrari, Elizabeth et al. (1993), Curitiba: Transição Pós-Moderna? Anais XVII ENANPAD, Salvador, ANPAD, p. 252-260.

FERrEIRA, Ana Luiza e Ricci, Ruda. (1992), "Estudos de Gestão: Ronda Alta e São João do Triunfo". POLIS Publicações, n.8, São Paulo, POLIS

Fischer, T. e Carvalho, J. (1993), "Poder Local, Redes Sociais e Gestão Pública”. In

Fischer, T. (Org) (1993), "Poder Local, Governo e Cidadania". Rio de Janeiro, Fundação Getúlio Vargas, 134-150.

_ et al. (1993), "Gestão Municipal, Descentralização e Participação: Um Estudo Comparado Espanha/Brasil”. In FISCHER, T. (Org), Poder Local, Governo e Cidadania. Rio de Janeiro, Fundação Getúlio Vargas, 115-123.

Fórum Nacional de Participação Popular nas Administrações Minicipaisa Democraticas (VVAA). (1994), Participação Popular nos Governos Locais. POLIS Publicações, n. 14, São Paulo, Polis.

FreEman, C. (1991), "Networks of Innovations: a Synthesis of Research Issues". Research Policy, North-Holand, (20): 499-514.

Guimarães, P. e D'ArC, R. (1994), "Participação Social e Administração Pública: Elementos para Discussão sobre Gestão das Cidades Brasileiras nos Anos 90". Encontro Cidadania e Movimentos Sociais, Salvador, CRH/UFBa, (mimeo)

HAMEL, Pierre. (1990), "Developpement Local: une nouvelle culture politique". Espaces Tempes. Paris, Association Espace Tempes, (43-44): 43-49.

HARVEY, David. (1989), "From Manegerialism to Entrepreneurialism: The Transformation in Urban Governance in Late Capitalisme". Estocolmo, Geografiska Annaler, (71b): 3-17.

Kowarick, Lucio e Singer, André. (1993), "A Experiência do Partido dos Trabalhadores na Prefeitura de São Paulo”. Novos Estudos, São Paulo, CEBRAP, (35): 195-216, mar/1993.

LEAL, Suely. (1994), Para alem do Estado: tenências, limites e alcance das novas formas de Gestão Urbana. Campinas, UNICAMP, tese de doutorado. 
Legales, P. e Parkinson, M. L (1994), "Inner City Policy En Grande Bretagne". Revue Française d'Administration Publique, Paris, (71): 483-498.

Levy, E.O (1992), "Impacto da Democracia nas Organizações Públicas Locais". Palestra proferida no V Colóquio sobre Poder Local. Salvador, NPGA/EAUFBa, (mimeo).

Lodovici, E. Samek e Bernareggi, G. M. ( 1992), Parceria PublicoPrivado: Cooperação Financeira e Organizacional entre o Setor Privado e Administrações Publicas Locais. São Paulo, Summus.

Lolola, E. e Moura, S. (1995), Análise de Rede(s): uma Contribuição aos Estudos Organizacionais. Salvador, NPGA/EAUFBa, (mimeo).

- (1994), Desenvolvimento Local e Economia Globalizada: onde estão as convergências? Salvador, NPGA/EAUFBa, (mimeo).

Massolo, A. (1988), "Em Direção às Bases: Descentralização e Município". Espaço e Debates, NERU, (24): 40-54.

Miller, H. "Post-Progressive Public Administration: Lessons From Policy Networks in Public Administration Review". American Society for Public Administration, Washington, 54(4): 378-385.

MorgenszTERn, Florence e Slama, Raphael (orgs). (1991), "Le Clair-Obscur des Reseaux". Revue POUR, Paris, GREP, (132).

Moura, Suzana. (1993), "Governo Local e Participação Popular: Ideário e Prática”. Panizzi, Wrana e Rovatti, João (orgs). "Estudos Urbanos: Porto Alegre e seus Planejamentos". Porto Alegre, Editora Universidade/Prefeitura Municipal, p.279-288.

_ (1993), "Inovações Municipais em Tempos de Democratização e Crise: Novos Cenários da Gestão Urbana”. Análise e Dados. Salvador, CEI, (1): 127-134, jun/1993.

_ (1994), "A Questão Publico-Privado: Antigos e Novos Sentidos". Anais XVIII ENANPAD, Curitiba, ANPAD.

- (1994), Pistas para o Estudo das Políticas Públicas no Contexto Atual. Salvador, NPGA/EAUFBa.

e Pinho, A. J. (1993), "Governos Locais em Contexto de Democratização e Crise". In Fischer, T. (org) Poder Local, Governo e Cidadania. Rio de Janeiro, Editora Fundação Getúlio Vargas, 291-308.

NoGueIRA, Heloisa et alli. (1992), "Experiências Inovadoras de Gestão Municipal”. POLIS Publicações, n. 9, São Paulo, POLIS.

Nogueira, Marco Aurélio. (1994), "Permanência e Mudança no Setor Publico". Revista do Setor Publico, Brasília, ENAP, (1): 43-54, jan/jul 1994.

NunES, Edson et alli. (1991), Município y Democracia en Ciudades Intermedias de América Latina. Santiago. Ediciones SUR.

Oliverra, C. A. (1995), Democracia nas Grandes Cidades: A Gestão Democrática da Prefeitura de Porto Alegre. Rio de Janeiro, IBASE.
Osborne, David e GaEbler, Ted. (1993), Reinventing Governement: How the Entrepeneurial Spirit is Transforming the Public Sector. New York, A Plume Book.

PACHECO, Regina. (1993), Iniciativa Econômica Local: a Experiência do ABC. LODOVICI, E. Samek e BERNAREGGI, G. M. Parceria Publico-Privado: Cooperaçao Financeira e Organizacional entre o Setor Privado e Administrações Públicas Locais. São Paulo, Summus, v.2, 1993.

_ (1994), "Estrategias de Desenvolvimento Local". Palestra proferida no VI Colóquio sobre Poder Local, Salvador. NPGA/EAUFBa, (em fita).

Pavía, P. et Ospina, C. (1992), Poder Local: Realidade y Utopia de la Descentralizacion en Colombia. Bogotá, Tercer Mundo Editores.

Penalva, Susana e Finquelievich, Susana. (1993), Innovative Experiences of Public and Private Sectors for Sustainable Development in Megacities: the case of Buenos Aires. Buenos Aires, CEUR, mimeo.

PInHANEZ et alli. (1993), Curitiba, uma Estratégia de Marketing. São Paulo, EAESP/FGV, mimeo.

RandolPh, R. (1993), "Redes Estratégicas e de Solidariedade". V Encontro Nacional da ANPUR, Belo Horizonte.

_ (1994), "Redes Estratégicas e Redes de Solidariedade: suas Escalas e seus Conflitos a nível Local". Palestra proferida no VI Colóquio sobre Poder Local, Salvador, NPGA/EAUFBa, (mimeo).

SCHERER-WARrEn, Ilse. (1994), "Metodologia de Redes para o Estudo das Organizações”. Palestra Proferida no VI Colóquio sobre Poder Local. Salvador, NPGA/EAUFBa, (em fita).

SiLva, Tarcilla L. da et alli. (1993), Governo Local Empreendedor em Curitiba: Mito ou Realidade? São Paulo, EAESP/FGV, mimeo.

STORPER, M. (1994), "Desenvolvimento Territorial na Economia Global do Aprendizado: o Desafio dos Países em Desenvolvimento". In Ribeiro, L.C. (org) "Globalização, Fragmentação e Reforma Urbana: o Futuro das Cidades Brasileiras”, São Paulo, Ed. Civilização Brasileira, 23-64.

SYRETT, Stephen. (1993), "Local Economic Iniciatives in Portugal: Reality and Rhetoric". International Journal of Urban and Regional Research. Oxford/Cambridge, Blackwel Publishers, 8-17 (4): 526-546.

Topalov, C. O (1993), "Encontro com o Local". Fischer, T. (org). Poder Local, Governo e Cidadania. Rio de Janeiro, Ed. Fundação Getúlio Vargas, 21-27.

Villassante, Tomas. (1989), Redes Comunitarias y Nuevas Cosmologías. ALFOZ, Madrid, p. 21-28.

_ (1995), "Clientelas y Emancipaciones: una Introducción Metodológica”. Villassante, T. (org). Las Ciudades Hablan. Caracas, Editorial Nueva Sociedad, 25-50. 


\section{RESUMEN}

Este artículo discute los cambios que están ocurriendo en la gestión local en Brasil, en las dos últimas décadas, tanto en términos de ideas como en la práctica. Se empieza identificando los movimientos que aparecen como propulsores de la innovación y algunas de las tendencias presentes en los años 90 de interacción gobierno sociedad.

\section{ABSTRACT}

This article discuses the changes which are happening in the local administration in Brazil, in the last two decades, both in the ideological and practical terms. It begins identifying the movements which appear as propellers of change and some of the present tendencies in the nineties of interrelation government-society.

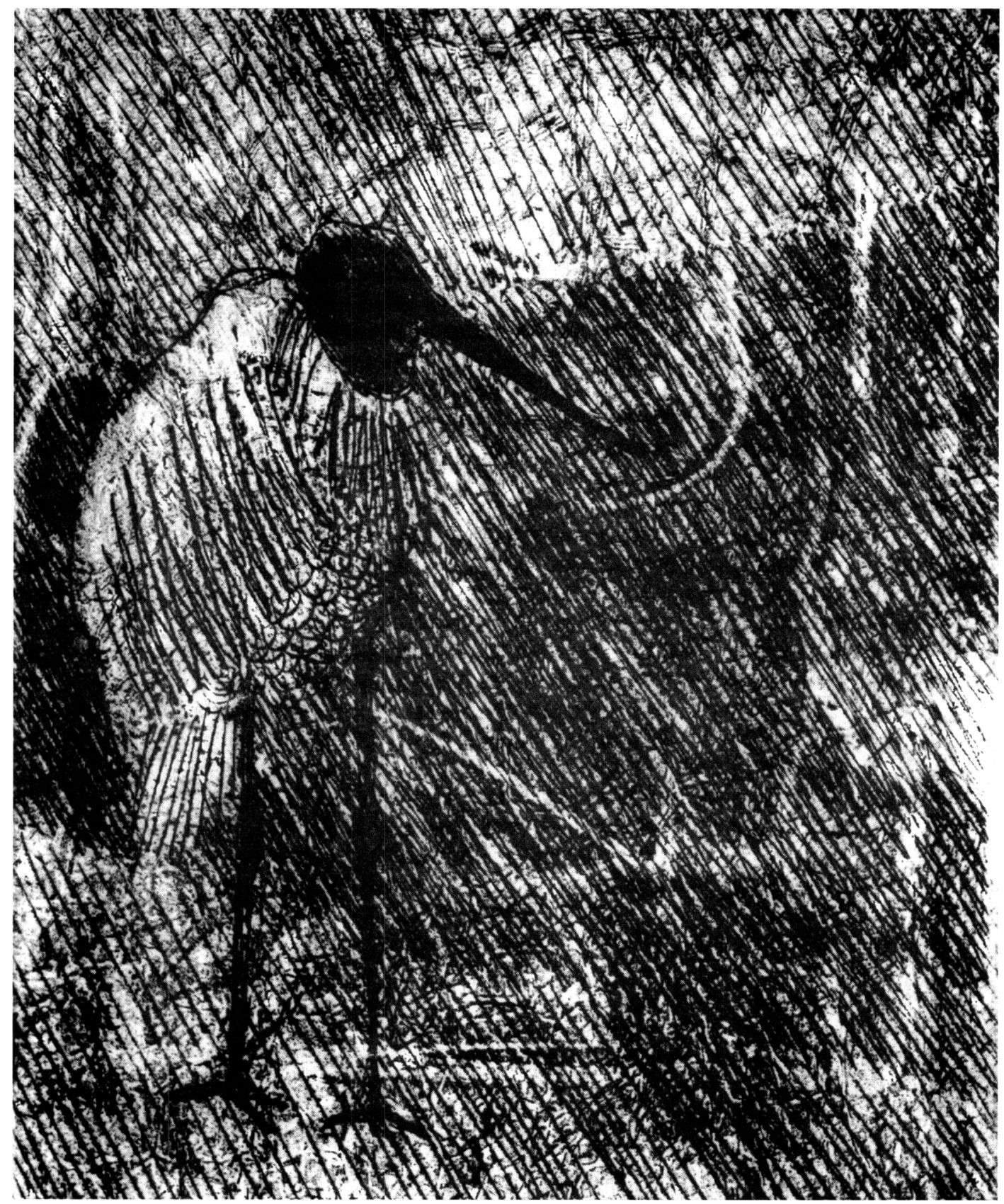

